\title{
Theoretical Aspect of Physical Phenomena in Inorganic Photovoltaic Cells. Electrical Modeling and Numerical Simulation.
}

\author{
Mohammed AZZA ${ }^{1 *}$, Jabran DAAIF ${ }^{1}$, Abd Elhadi CHAHID ${ }^{3}$, Mohammed SALAH ${ }^{2,4}$, Said BELAAOUAD ${ }^{1}$ \\ ${ }^{1}$ Laboratory Physical Chemistry of Materials, Faculty of Sciences Ben M'Sik, University Hassan II, Casablanca, Morocco. \\ ${ }^{2}$ Team of Chemoinformatics Research and Spectroscopy and Quantum Chemistry, Physical and Chemistry Lab, Faculty of Science, \\ University Chouaib Doukkali, B. P. 20, 2300, El Jadida, Morocco. \\ ${ }^{3}$ Regional Center for Education and Training CRMEF-Beni-Mellal Khenifra. Mohamed V Street. BeniMellal,. Morocco. \\ ${ }^{4}$ LS3MN2E, CERNE2D, Department of Chemistry, Mohammed V University, Faculty of Sciences Rabat, Morocco
}

\begin{abstract}
This work is based on the development of a theoretical model describing the drift and diffusion transport of photogenerated charge carriers and the impact of space charge on this transport in relation to the different physical phenomena characterizing the photovoltaic conversion in an inorganic silicon-based cell. In a second step, we used a numerical solution of the transport differential equations based on the Runge-Kutta algorithm in the framework of the finite difference method, This led us to an electrical model of the photovoltaic cell and of the photo-generated currents by RLC circuit equipped with a diode modeling the direction of electron and hole transport and allowed us to study the relations between the optical and electrical properties of the cell, as well as the influence of the different concentrations of impurities used for the n-type and p-type doping of the silicon on the properties of absorption of the light photons, the spectral response as well as the conductivity, the open-circuit potential and the short-circuit current.
\end{abstract}

Keywords: numerical simulation; electrical modeling; finite difference method; solar cell

\section{Introduction}

The absorption of light photons by a semiconductor leads to the photo-generation of charge carriers, electrons and holes, through the formation of excitons which are considered as bound states of an electron and a hole, the photovoltaic effect [1-2]. The dissociation of these excitons under the influence of the electric field of the $\mathrm{p}-\mathrm{n}$ junction leads to the generation of electrons and holes which diffuse from the p-n junction towards the electrode-semiconductor contact zones and thus their collection on the cathode for electrons and on the anode for holes. This transport phenomena are due to the concentration and junction potential gradients, the transport equations describing the main physical phenomena related to photovoltaic conversion and charge carrier diffusion are derived from the differential equations related to the electron and hole current densities by calculating the divergence of the current density vectors and using the kinetic evolution of charge densities [5-6].The effect of the space charge zone on the generation and recombination phenomena as well as on the transport of the load carriers is also considered. The study of the hope charge zone is based on the Nernst-Planck equations, the transport equations and the Poisson equation.[7] These equations allowed us to establish the theoretical model on which the numerical simulation of the cell is based, using the Runge-Kutta algorithm derived from the finite difference method for solving the differential equations obtained. On the other hand, we proceeded to model the silicon-based inorganic photovoltaic cell by an equivalent circuit focusing on the generated photo-currents and their relationship with the densities of the donor and acceptor impurities, the wavelength, the open circuit potential and the luminous flux, In a second row, we have also approached the resistances equivalent to the series, source and shunt resistances by trying to elucidate with maximum clarity, the links between the structural properties of the material at the microscopic scale with the optical and electrical properties of the photovoltaic cell.[8-9].

\section{Theoretical model}

\subsection{Diffusion and drift of electrons and holes}

For the electron:

$$
\vec{J}_{e}=\left[-q D_{e} \frac{\partial n e}{\partial x}+q n_{e} \mu_{e} \frac{\partial \phi}{\partial x}\right] \vec{\imath}
$$

*Corresponding author:mohammedazza81@gmail.com 
For the holes:

$$
\vec{J}_{h}=\left[-q D_{h} \frac{\partial n_{h}}{d x}-q p \mu_{h} \frac{\partial \phi}{\partial x}\right] \overrightarrow{\mathbf{l}}
$$

\subsubsection{Continuity equations relating to electrons and holes}

Let us calculate the one-dimensional divergence of the two vector fields $\vec{J}_{\mathrm{h}}$ and $\overrightarrow{\mathrm{J}}_{\mathrm{e}}$ in order to arrive at the diffusion and drift equations relating to the charge carriers:[10].

$$
\operatorname{divJ}_{e}=\frac{\partial J_{e}}{\partial x}=+q \frac{\partial}{\partial x}\left[-\frac{\partial n_{e}}{\partial x}+n_{e} \mu_{e} \frac{\partial \phi}{\partial x}\right]
$$

This therefore allows us to arrive at the expression of the diffusion equation and the drift relating to the electrons

$$
\begin{array}{r}
\frac{1}{q} \frac{\partial J_{e}}{\partial x}=-\frac{\partial D_{e}}{\partial x} \frac{\partial n_{e}}{\partial x}-D_{e} \frac{\partial^{2} n_{e}}{\partial x^{2}}+\mu_{e} \frac{\partial n_{e}}{\partial x} \frac{\partial \phi}{\partial x} \\
+n_{e} \frac{\partial \mu_{e}}{\partial x} \frac{\partial \phi}{\partial x}+n_{e} \mu_{e} \frac{\partial^{2} \phi}{\partial x^{2}}
\end{array}
$$

For $D_{e}=$ cte et $\mu_{e}=$ cte

The continuity equation reduces to:

$$
\frac{1}{q} \frac{\partial J_{e}}{\partial x}=-D_{e} \frac{\partial^{2} n_{e}}{\partial x^{2}}+\mu_{e} \frac{\partial n_{e}}{\partial x} \frac{\partial \phi}{\partial x}+n_{e} \mu_{e} \frac{\partial^{2} \phi}{\partial x^{2}}
$$

The same for the holes gives:

$$
\frac{1}{\mathrm{q}} \frac{\partial \mathrm{J}_{\mathrm{h}}}{\partial \mathrm{x}}=-\mathrm{D}_{\mathrm{h}} \frac{\partial^{2} \mathrm{n}_{\mathrm{h}}}{\partial \mathrm{x}^{2}}-\mu_{\mathrm{h}} \frac{\partial \mathrm{n}_{\mathrm{h}}}{\partial \mathrm{x}} \frac{\partial \phi}{\partial \mathrm{x}}-\mu_{\mathrm{h}} \mathrm{n}_{\mathrm{h}} \frac{\partial^{2} \phi}{\partial \mathrm{x}^{2}}
$$

The charge carriers diffuse from the (p-n) junction to the cathode for electrons and to the anode for holes.

\subsubsection{Gauss -Poisson Equation}

The Gauss-Poisson equation is an ecliptic equation deduced from the laws of electrostatics, for intrinsic one-dimensional semiconductors this equation takes the form [11-14]:

$$
\frac{\partial^{2}}{\partial x^{2}} \phi(x)=-\frac{q}{\varepsilon}\left(n_{h}-n_{e}\right)
$$

For extrinsic semiconductors provided with ionized sites (impurities) and structural defects, we can write [12].

$$
\begin{gathered}
\frac{\partial^{2} \phi(\mathrm{x})}{\partial x^{2}}+\frac{q}{\varepsilon}\left(n_{h}-n_{e}+N_{A^{-}}+N_{D^{+}}+n_{t}\right) \\
=0
\end{gathered}
$$

\subsubsection{Determination of the expression of the junction electric field}

By integrating the Nernst -Planck equations relating to the densities of electronic currents and those of holes we find as an expression for the junction electric field In the case of an open circuit

$$
E_{\text {int }}=-\frac{k T}{q} \frac{\mu_{e} \frac{d n_{e}}{d x}-\mu_{h} \frac{d n_{h}}{d x}}{n_{e} \mu_{e}+n_{h} \mu_{h}}
$$

It is the junction electric field which appeared during the irradiation of the material whose electric neutrality is no longer respected; this field is due to the diffusion currents which appeared under the influence of the concentration gradients of the carriers of load out of equilibrium when irradiation is not uniform.

This permits to write the Poisson equation:

$$
\frac{d^{2} \phi}{d x^{2}}-\frac{k T}{q} \frac{\mu_{e} \frac{d^{2} n_{e}}{d x^{2}}-\mu_{h} \frac{d^{2} n_{h}}{d x^{2}}}{n_{e} \mu_{e}+n_{h} \mu_{h}}=0
$$

By solving the Poisson equation we find:

$$
\phi=\frac{K T}{q} \int \frac{d n_{e} \mu_{e}-\mu_{h} d n_{h}}{\mu_{e} n_{e}+\mu_{h} n_{h}}
$$

Therefore:

$$
\begin{aligned}
\frac{q}{\varepsilon}\left[n_{h}-n_{e}+N_{D^{+}}\right. & \left.+N_{A^{-}}+n_{t}\right] \\
+ & \frac{k T}{q} \frac{\mu_{e} \frac{d^{2} N}{d x^{2}}-\mu_{h} \frac{d^{2} N}{d x^{2}}}{N}=0
\end{aligned}
$$

Where $\mathrm{N}$ is the total density:

$$
\mathrm{N}=\mathrm{n}_{\mathrm{h}}-\mathrm{n}_{\mathrm{e}}+\mathrm{N}_{\mathrm{D}^{+}}+\mathrm{N}_{\mathrm{A}^{-}}+\mathrm{n}_{\mathrm{t}}
$$

The absorption of the radiation gives rise to a process of photoionization and causes an increase in the energy of the electrons and holes which remain bound and form an exciton when the concentration of the ND donor centers of low ionization energy is large enough ; here is overlap of the orbitals of the valence band and the conduction band which leads to a transfer of electrons by tunnel effect when the donor material is subjected to the action of an external electric field $\vec{E}_{\text {ext }}$, these transitions electronics give rise to conduction by jumps, this is the phenomenon of photoconductivity.

\subsection{The space charge}

In the case of unipolar conductivity $\left(\mathrm{n}_{\mathrm{h}}=0\right)$ we write the electric field by virtue of the Poisson equation:

$$
\frac{d E(x)}{d x}=-\frac{K T}{q} \frac{1}{n} \frac{d^{2} n}{d x^{2}}
$$

From where: $E(x)=-\frac{K T}{q} \frac{1}{n} \frac{d n}{d x}$

So:

$$
\phi(x)=\frac{K T}{q} \ln \frac{n(x)}{n_{0}}
$$

It's about Nernst potential

$$
n_{e}(x)=n_{0} \exp \left(-\frac{q \phi(x)}{K T}\right)
$$

For the holes we have $n_{h}(x)=n_{0} \exp \left(\frac{q \phi(x)}{k T}\right)$

Densities follow the Maxwell-Boltzmann distribution law by substituting the coordinate $\mathrm{x}$ by the spherical coordinate. The electric charge density will then be:

$$
\rho(r)=q n_{i}(r)=q n_{i} \exp \frac{-q \phi(r)}{k T}
$$

and by virtue of the expression of $\phi(r)$

$$
\rho(r)=\mathrm{qn}_{\mathrm{i}} \exp -\frac{\mathrm{q}^{2}}{4 \pi \varepsilon r \mathrm{kT}}
$$


Since the electrostatic interactions are relegated to thermal agitation therefore we have $\mathrm{q} \phi \ll<\mathrm{kT}$, the expansion of the exponential gives as an expression of $n$ (r) by virtue of the make that $n_{e}=n_{h}$

$$
n_{i}(r)=-\frac{q^{2} n_{i}}{4 \pi \varepsilon r k T}
$$

And the nonlinear equation of $\phi(r)$ in spherical coordinates is linearized to take the form

$$
r^{2} \frac{d^{2} \phi}{d r^{2}}+2 r \frac{d \phi}{d r}+\frac{n_{i} q^{2} r^{2}}{k T \varepsilon} \phi=0
$$

This admits for the solution

$$
\phi=\frac{q}{4 \pi \varepsilon r} \exp ^{-}\left(\frac{n_{i} q^{2}}{k T \varepsilon}\right)^{1 / 2} r
$$

Let's pose:

$\mathrm{l}_{\mathrm{D}}=\sqrt{\frac{k T \varepsilon}{n_{i} q^{2}}}:$ The thickness of the space charge zone (Debye length)

$\mathrm{l}_{\mathrm{D}} \approx 1 A^{\circ}:$ it is the characteristic length of the space charge in the proximity of two regions containing different densities of charge carriers.

So:

$$
\phi=\frac{q}{4 \pi \varepsilon r} \exp ^{-\frac{r}{l_{D}}}
$$

$$
\text { If } r \ll l_{D} \Rightarrow \phi=\frac{q}{4 \pi \varepsilon}\left(1-\frac{r}{l_{D}}+\frac{r^{2}}{l^{2}{ }_{D}}+\cdots\right)
$$

So $\phi \approx \frac{q}{4 \pi \varepsilon r}-\frac{q}{4 \pi \varepsilon l_{D}}$

In thermodynamic

$$
\begin{aligned}
W_{e}=-\int_{0}^{\phi} q d \phi & \\
& =-\frac{q^{2}}{8 \pi \varepsilon}\left(\frac{1}{r}\right. \\
& \left.-\frac{1}{l_{D}}\right)
\end{aligned}
$$

From the laws of thermodynamics, the dissociation energy of the excitons is related to the dissociation equilibrium constant

$$
-R T \ln K_{e D}=-\frac{q^{2} N_{A}}{8 \pi \varepsilon}\left(\frac{1}{r}-\frac{1}{l_{D}}\right)
$$

That is $\quad \ln \mathrm{K}_{\mathrm{eD}}=\frac{\mathrm{q}^{2}}{8 \pi \varepsilon \mathrm{kT}}\left(\frac{1}{\mathrm{r}}-\frac{1}{\mathrm{l}_{\mathrm{D}}}\right)$

It is therefore the dissociation constant of the excitons at equilibrium taking into account the space charge zone; the electric double layer reigning in this zone favors even more the dissociation of the excitons; the potential drop due to the space charge.

$$
\Delta \phi=\frac{\mathrm{ql}_{\mathrm{D}}^{2}}{2 \varepsilon}\left(\mathrm{n}_{\mathrm{h}}-\mathrm{n}_{\mathrm{e}}+\frac{1}{\frac{1}{\mathrm{~N}_{\mathrm{D}}}-\frac{1}{\mathrm{~N}_{\mathrm{A}}}}\right) \Delta \phi<\frac{\mathrm{KT}}{\mathrm{q}}
$$

The drop in potential becomes negligible the current limit by the space charge is given by the Mott-Gurney law:

$$
\begin{gathered}
J=\frac{9}{8} \frac{\mu \varepsilon}{l_{D}^{3}}\left(\phi_{b i}-V\right)^{2} \\
x=l_{D} \text { et } \quad x=-l_{D} \\
\text { On a } \frac{d \phi}{d x}=0
\end{gathered}
$$

$-l_{D}<x<0: \rho=-q N$ p-type conduction

$0<x<l_{D} \quad: \rho=+q N$ n-type conduction

$$
\frac{\partial J_{n_{e}}}{\partial x}+\frac{\partial J_{n_{h}}}{\partial x}=0
$$

$$
J_{n_{e}}+J_{n_{h}}=0
$$

With $\mathrm{Jn}=0$ at the acceptor-cathode interface and Jp $=0$ at the anode-donor interface, because $\mathrm{p}>\mathrm{n}$ and because $\mu \mathrm{e}>\mu \mathrm{h}$ we then have $V_{\text {illu }} \neq V_{\text {obs }}$ and this is due to the space charge, there is therefore accumulation of charge

\section{Method for numerical resolution of differential equations (numerical modeling)}

The equations obtained are non-linear and strongly coupled equations so they do not admit analytical solutions except in very simplified cases.

The method used for the numerical solution approximation analysis of these differential equations of diffusion and drift for electrons and holes is the RungeKutta method because of his simplicity, its ease and its adequacy for the numerical resolution of conservation equations for physical systems with simple geometry.[11]

The adopted meshes are:

$$
\begin{aligned}
& \{x(i)=(i-1) \Delta x\} \\
& \{t(j)=(j-1) \Delta t\}
\end{aligned}
$$

These are steps of discretization; $\mathrm{i}$ and $\mathrm{j}$ are the indices of discretization.

\section{Results and discussion}

A dimensional writing of the diffusion equation in the form of a Boltzmann kinetic equation

$$
\frac{d^{2} n^{*}}{d \eta^{2}}+2 \eta D^{*^{2}} \frac{d n}{d \eta}+\left(n^{*} \frac{d n^{*}}{d \eta}\right)=0
$$

\subsection{Discretization of the exciton diffusion equation in space and time}

$$
D_{E} \frac{\partial^{2} \rho_{E}}{\partial x^{2}}-\frac{\partial_{\rho E}}{\partial t}+\frac{\rho_{E}}{\tau}-G_{E}
$$

The application of the discretization operator

$$
\begin{array}{r}
{\left[\hat{d} s ; \hat{\Delta} \rho_{E}\right]=\frac{\rho(E)_{(i+1)}-2 \rho_{E(i)}+\rho_{E}(i-1)}{\Delta x^{2}}} \\
{\left[\hat{d} ; \hat{\partial}_{t} \rho_{E}\right]=\frac{\rho_{j+1}^{(E)}-\rho_{j-1}^{(E)}}{2 \Delta t}}
\end{array}
$$


So differential equation (26) can be written as the numerical difference equation as follows:

$$
\begin{gathered}
\frac{\rho_{i+1}-2 \rho_{i}+\rho_{i-1}}{\Delta x^{2}}-\frac{\rho_{j+1}-\rho_{j-1}}{2 \Delta t}=-\frac{1}{D_{E}}\left(\frac{\rho_{E}}{\tau}-G_{E}\right) \\
J_{e}=-q D_{e} \frac{\partial n_{e}}{\partial x}+q n_{e} \mu_{e} \frac{\partial \phi}{\partial x} \\
\boldsymbol{J}_{\boldsymbol{e}}=-\boldsymbol{q} \boldsymbol{D}_{e} \frac{\partial \boldsymbol{n}_{e}}{\partial x}+\boldsymbol{q} \boldsymbol{n}_{e} \boldsymbol{\mu}_{e}
\end{gathered}
$$

In stationary regime we can write

$$
-2 \rho_{i}+\rho_{i-1}=-\frac{1}{D_{E}}\left(\rho_{E}-G_{E}\right) \Delta x^{2}
$$

The method followed here is that of Runge-Kutta of the second order which requires two iteration steps of the system of implicit equations:[12-13]

$$
\begin{gathered}
t=0 \rightarrow t=j+\frac{1}{2} \\
t=j+\frac{1}{2} \rightarrow t=j+1 \\
\rho_{E}^{j+\frac{1}{2}}=\rho_{E}^{j}+\frac{\Delta t}{2}\left[\frac{D_{E}\left(\rho_{i+1}-2 \rho_{i}+\rho_{i-1}\right.}{\Delta x^{2}}\right]
\end{gathered}
$$

Based on equation 27 we find:

$$
\rho_{j+1}=\rho_{j-1}+2 \Delta t\left[\frac{D_{E}\left(\rho_{i+1}-2 \rho_{i}+\rho_{i-1}\right)}{\Delta x^{2}}+\frac{\rho_{E}}{\tau}-G_{E}\right]
$$

\subsection{Discretization of the current density equation}

In adimensional writing the equations take below the form:

$$
J^{*}=-D^{*} \frac{d n^{*}}{d \eta^{*}}+n^{*} \mu^{*} \frac{d U^{*}}{d \eta}
$$

This equation can be written as a system of two equations:

$$
\begin{gathered}
J_{e}^{*}(m i g)=n^{*} \mu_{e}^{*} \frac{d U^{*}}{d \eta} \\
J_{e(d i f f)}^{*}=-D^{*} \frac{d n^{*}}{d \eta}
\end{gathered}
$$

The application of the discretization operator results in the following differences equations:

$$
\begin{aligned}
& \frac{\mathrm{dU}^{*}}{\mathrm{~d}^{*}}=\frac{\mathrm{U}_{\mathrm{i}+1}^{*}-\mathrm{U}_{\mathrm{i}-1}^{*}}{2 \Delta \eta} \\
& \frac{\mathrm{dn}^{*}}{\mathrm{~d} \eta}=\frac{\mathrm{n}_{\mathrm{i}+1}^{*}-\mathrm{n}_{\mathrm{i}-1}}{2 \Delta \eta}
\end{aligned}
$$

So :

$$
\begin{gathered}
J_{e}^{*}=\frac{n^{*} \mu^{*}}{2 \Delta \eta}\left[\left(U_{i+1}^{*}-U_{i-1}^{*}\right)-D^{*}\left(n_{i+1}^{*}-n_{i-1}^{*}\right)\right] \\
\left.n_{i+1}=n_{i-1}+\frac{1}{D^{*}}\left[U_{i+1}-U_{i-1}\right)-\frac{2 \Delta \eta}{n^{*} \mu^{*}} J^{*}\right]
\end{gathered}
$$

\subsection{Discretization of the charge conservation equation}

$$
\begin{gathered}
\frac{1}{q} \frac{\partial J}{\partial \eta^{*}}=-D \frac{d^{2} n^{*}}{d \eta^{* 2}}+\mu \frac{d U^{*} d n^{*}}{d \eta^{*} d \eta^{*}}+\frac{n \mu}{d \eta^{* 2}} d^{2} U^{*} \\
\frac{\partial n}{\partial t}-G+R=\frac{1}{q} \frac{\partial J}{\partial \eta^{*}} \\
\frac{\partial n^{*}}{\partial t}-\alpha(\lambda)\left(1-r(\lambda) \phi(\lambda) \exp ^{-\alpha(\lambda) \eta^{*}-\frac{\Delta n^{*}}{\tau}}\right. \\
=-D^{*} \frac{\partial^{2} n^{*}}{\partial \eta^{* 2}}+\mu^{*} \frac{d U^{*}}{d \eta^{*}} \\
+n^{*} \mu^{*} \frac{d^{2} U}{d \eta^{* 2}}
\end{gathered}
$$

Let's now move on to the difference equations and application of the discretization operator:

$$
\begin{gathered}
{\left[\hat{d}_{s} ; \partial_{t} n^{*}\right]=\frac{n_{j+1}^{*}-n_{j-1}^{*}}{2 \Delta t}} \\
{\left[\hat{d}_{s} ; \frac{d^{2} n_{e}^{*}}{d \eta^{2}}\right]=\left[\hat{d}_{s} ; \hat{\Delta} n_{e}\right]=\frac{n_{e(i+1)}-2 n_{i}^{e *}+n_{i-1}^{e *}}{2 \Delta \eta^{*^{2}}}}
\end{gathered}
$$

as well as $\frac{d U^{*}}{d \eta^{*}}=\frac{U^{*}{ }_{i+1}-U^{*}{ }_{i-1}}{2 \Delta \eta^{*}}$ avec

$$
\begin{aligned}
\Delta \eta^{*}=\eta_{i+1}^{*}- & \eta_{i-1}^{*} \\
\frac{d n_{e}^{*}}{d \eta^{*}} & =\frac{n_{e_{(i+1)}}-n_{e(i-1)}}{2 \Delta \eta^{*}}
\end{aligned}
$$

Which finally permits us to write?

$$
\begin{aligned}
\frac{n_{j+1}^{(e *)}-n_{j-1}^{(e *)}}{2 \Delta t}- & \alpha(\lambda)(1-R(\lambda)) \phi(\lambda) \exp ^{-\alpha(\lambda) \eta^{*}} \\
& -\frac{n_{e(j+1)}-n_{e(j-1)}}{\tau} \\
& =-\frac{D^{*}\left(n_{e(j+1)}-2 n_{e(i)}+n_{e(i-1)}\right.}{2 \Delta \eta^{*^{2}}} \\
& +\mu_{e}^{*} \frac{\left(U_{i+1}^{*}-U_{i-1}^{*}\right)}{2 \Delta \eta^{*}} \frac{n_{i+1}-n_{i-1}}{2 \Delta \eta^{*}} \\
& +n_{e} \mu_{e} \frac{\left(U_{i+1}-2 U_{i}+U_{i-1}\right)}{2 \Delta \eta^{*}}=0
\end{aligned}
$$

For the permanent regime $\frac{n_{(j+1)}^{(*)}-n_{(j-1)}^{(*)}}{2 \Delta t}=0$

This means:

$$
\begin{aligned}
n_{(i+1)}^{*}=n_{(i-1)}^{*}+ & \frac{2 D}{\mu_{e}^{*}\left(U_{i+1}^{*}-U_{i-1}^{*}\right)}\left[n_{(i+1)}^{*}-2 n_{(i)}^{*}\right. \\
& \left.+n_{(i-1)}^{*}\right] \\
& +\frac{2}{\mu\left(U_{i+1}^{*}-U_{i-1}^{*}\right)}[-\alpha(\lambda)(1 \\
& \left.-R(\lambda)) \phi(\lambda) e^{-\alpha(\lambda) \eta^{*}}-\frac{n}{t}\right] \Delta \eta^{2 *} \\
& -\frac{n\left(U_{i+1}^{*}-2 U_{i}^{*}+U_{i-1}^{*}\right)}{\left(U_{i+1}^{*}-U_{i-1}^{*}\right)}
\end{aligned}
$$

\subsection{Discretization of the Poisson equation}

$$
\frac{\partial^{2 U^{*}}}{\partial \eta^{*^{2}}}=-\frac{q}{\varepsilon}\left(n_{h}-n_{e}+N^{+}+N^{-}+n_{\text {piéges }}\right)
$$

Let's apply the operator to the differences $\widehat{\mathrm{d}}_{\mathrm{s}} \mathrm{sur} \Delta^{*} \mathrm{U}^{*}$

$$
\left[d_{s} ; \Delta^{*} U^{*}\right]=\frac{U_{i-1}^{*}-2 U_{I}+2 U_{i+1}}{2 \Delta \eta^{*^{2}}}
$$

$$
\begin{aligned}
& \frac{U_{i-1}^{*}-2 U_{i}+U_{i+1}}{2 \Delta \eta^{*^{2}}} \\
&=\frac{-q}{\varepsilon}\left[n_{h}-n_{e}+N^{+}+N^{-}+n_{\text {pie }}\right]
\end{aligned}
$$




$$
\begin{aligned}
& U_{i+1}-2 U_{i}-U_{i-1} \\
& =-\frac{2 q}{\varepsilon}\left[n_{h}-n_{e}+N^{+}+N^{-}\right. \\
& \left.+n_{\text {piege }}\right] \Delta \eta^{2}
\end{aligned}
$$

Let us apply the operator of discretization only on the operator of the first derivation

$$
\begin{aligned}
& \frac{d}{d_{\eta}}\left[\frac{d}{d_{\eta}} U\right]=\frac{d}{d_{\eta}}\left[\frac{U_{i+1}-U_{i-1}}{\Delta \eta}\right] \\
& \frac{d}{d_{\eta}}\left[\frac{U_{i+1}-U_{i-1}}{\Delta \eta}\right]=\frac{-q}{\varepsilon}\left[n_{h}-n_{e}+N^{+}+N^{-}\right. \\
& \left.+n_{\text {pie }}\right] \\
& \frac{U_{i+1}^{*}-U_{i-1}}{\Delta \eta}=\frac{-q}{\varepsilon}\left[n_{h}-n_{e}+N^{+}+N^{-}\right. \\
& \left.+n_{\text {piege }}\right] d \eta \\
& =-\frac{1}{\varepsilon}\left[\sigma_{n_{h}}-\sigma_{n_{e}}+\sigma_{N^{+}}+\sigma_{N^{-}}\right. \\
& \left.+\sigma_{\text {piege }}\right] \\
& U_{i+1}=U_{i-1}+\frac{1}{\varepsilon}\left[\sigma_{n_{e}}-\sigma_{n_{h}}-\sigma_{N^{+}}-\sigma_{N^{-}}\right. \\
& \left.-\sigma_{\text {pieges }}\right] \Delta \eta^{*}
\end{aligned}
$$

In the space charge zone the diffusion potential takes the form:

$$
\phi_{\text {diff }}=\frac{K T}{q} \ln \frac{N_{A} N_{D}}{n_{i}^{2}}
$$

For the Zone of contact $\mathrm{x}=0$ we have

And the current density

$$
S_{n} \Delta n=D_{n} \frac{\partial n}{\partial x} \mid x=0
$$

$$
J_{n}=-q D_{n} \frac{\partial n}{\partial x} \mid x=0
$$

At the limit of the junction

$$
n=n_{0}=
$$

$$
\frac{n_{i}^{2}}{N_{A}} \mid x=x_{p}
$$

And the currentdensity

$$
J_{n_{h}}=-q D_{n_{h}} \frac{\partial n_{h}}{\partial x} \mid x=x_{n_{h}}
$$

\subsection{Electrical modeling and simulation of the photovoltaic cell}

\subsubsection{Photo-currents generated}

The generated photo-current is the sum of three currents. $\mathrm{J}_{\mathrm{e}}(\lambda)_{\text {diff }}$ Due to the transition of electrons from the valence band of the donor to the conduction band therefore to the drift of the electrons towards the p-type region

$\mathrm{J}_{\text {scz }}$ (pnjunction ) due to the dissociation of excitons and charge separation in the space charge zone.

$\mathrm{J}_{\mathrm{h}}(\lambda)$ : the current due to drift from the holes towards region $\mathrm{n}$ (relative to the acceptor)

$$
J_{p h}(\lambda)=J_{e}(\lambda)+J_{s c z}(\lambda)+J_{h}(\lambda)
$$

The current generated from the space charge zone is the sum of two currents

$$
J_{s c z}=J_{e}+J_{h}
$$

The total photo-current relating to the entire solar spectrum can be written

$$
J_{p h}(\lambda)=\int_{\lambda_{m}}^{\lambda_{M}} J_{p h}(\lambda) d \lambda
$$

In darkness; the current density is expressed by Shockley's law

$$
J=J_{0}\left[\exp \left(\frac{q V}{K T}\right)-1\right]
$$

Where $\mathrm{J}_{0}$ represents the saturation current density which has the form in the case of $\mathrm{p}$-n junction

$$
\begin{aligned}
& J_{0}=\frac{q n_{i}^{2} D_{h}}{N_{D} L_{h}} \\
& \text { With } \quad D_{h}=\frac{L_{h}^{2}}{\tau_{h}}
\end{aligned}
$$

We can write $J_{0}=\frac{q n_{i}^{2} L_{h}}{N_{D} \tau_{h}}$

Express $J_{0}$ as a function of the diffusional mobility of the holesBy virtue of the Nernst-Einstein equation we can write $: \frac{L_{h}^{2}}{\tau_{h}}=\frac{k T}{q} \mu_{h}$

$J_{0}$ Takes the form:

$$
J_{0}=\frac{K T n_{i}^{2}}{N_{D} L_{h}} \mu_{h}
$$

After illumination; the absorption of photons generates a photo-current $\mathrm{J}_{\mathrm{ph}}$ in such a way that the density of the total current becomes:

$$
J=J_{p h}-J_{0}\left[\exp \left(\frac{q V}{K T}\right)-1\right]
$$

$$
J=J_{p h}-\frac{K T n_{i}^{2}}{N_{D}} \frac{\mu_{h}}{L_{h}}\left[\exp \left(\frac{q V}{K T}\right)-1\right]
$$

The variation of the total current as a function of the ND impurities is represented by the following graph:

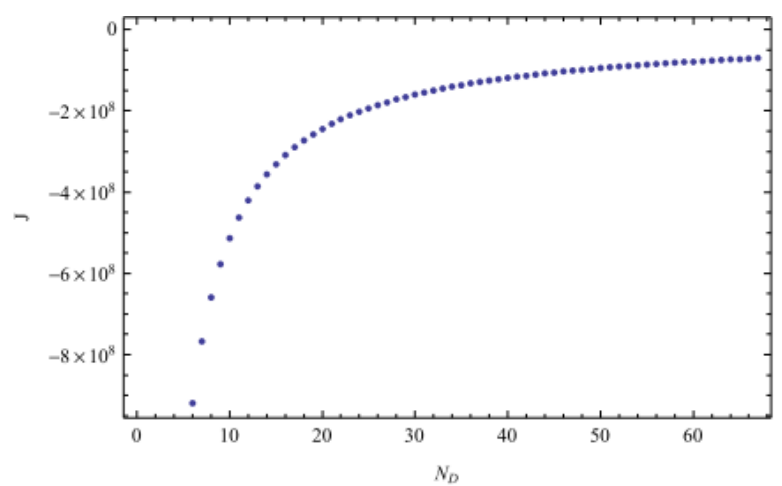

Fig. 1. The variation of the total current density as a function of the density of the donor impurities 
The total current increases rapidly with the density of the donor impurities and this is due to the decrease in dark current with the doping this results in the strong dependence of the total current mainly on the photocurrent. the doping This type increases the photoinduced electron donor sites and further decreases the dark current density.

This is the equation of the curve $\mathrm{J}(\mathrm{V})$ of the photovoltaic cell .At short-circuit that is to say at $\mathrm{V}=0$, the current density equals the density of the short-circuit current Jcc which is expressed as a function of luminous flux $\Phi$ in the form:

$$
J_{c c}=\frac{q \lambda}{h c} \Phi(\lambda)(1-r(\lambda)) P_{\text {diff }}(\text { exciton })
$$

$$
J_{c c}=K \Phi(\lambda)
$$

With $\mathrm{K}$ the photovoltaic sensitivity and $\mathrm{r}(\lambda)$ the spectral reflection coefficient:

$$
r(\lambda)=\left(\frac{\sqrt{\varepsilon_{r}(\lambda)}-1}{\sqrt{\varepsilon_{r}}+1}\right)^{2}
$$

Depending on the photovoltaic sensitivity we can express the quantum efficiency of the cell

$$
Q_{E}=\frac{J_{c c}}{\Phi(\lambda)} \frac{h c}{\lambda q}=\frac{K h c}{\lambda q}
$$

\subsubsection{Open-circuit voltage:}

Now let's determine the open circuit potential: We write $\mathrm{J}=0$

$$
V_{o c}=\frac{K T}{q} \ln \left(\frac{J_{c c}}{J_{0}}+1\right)
$$

Let us replace $\mathrm{J}_{0}$ by its expression:

$$
V_{o c}=\frac{K T}{q} \ln \left[\frac{N_{D} L_{h}}{K T n_{i}^{2} \mu_{h}} J_{c c}+1\right]
$$

As a function of the diffusion coefficient and the electronic mobility we find Knowing that:

$$
: \frac{D_{e}}{\mu_{e}}=\frac{D_{h}}{\mu_{h}}
$$

so:

$$
\mu_{h}=\mu_{e} \frac{D_{h}}{D_{e}}
$$

We can then write

$$
V_{o c}=\frac{k T}{q} \ln \left[\frac{N_{D} L_{h} D_{e}}{K T \mu_{e} D_{h} n_{i}^{2}} J_{c c}+1\right]
$$

For $J_{c c} \ll J_{0}$

We can write: $\mathrm{V}_{\text {oc }}=\frac{K T}{q} \frac{J_{c c}}{J_{0}}$
That is to say: $V_{o c}=\frac{K T}{q}\left[\frac{q \lambda}{h c J_{0}} \Phi(\lambda)\left(1-r(\lambda) p_{\text {diff }}^{E x}\right]\right.$

Si $J_{c c} \gg J_{0}$

$$
V_{o c}=\frac{K T}{q} \ln \frac{q \lambda \Phi(\lambda)[1-r(\lambda)] p_{\text {diff }}^{E}}{h c J_{0}}
$$

\subsubsection{Energy efficiency of the cell:}

This efficiency is dependent on the quantum efficiency by

$$
E_{E}=\frac{q \lambda}{h c} Q_{E} V_{o c}
$$

Where QE is the quantum efficiency of the photovoltaic conversion the spectral wavelength response of the photovoltaic cell depends more often on the associated electrical circuit has the form

$$
R_{s p}(\lambda)=\frac{J_{p h}(\lambda)}{q \Phi(\lambda)(1-r(\lambda))}
$$

By virtue of the dependency of photo-current on Voc input, the variation of this spectral response in relation to the open circuit potential is illustrated by the graph below

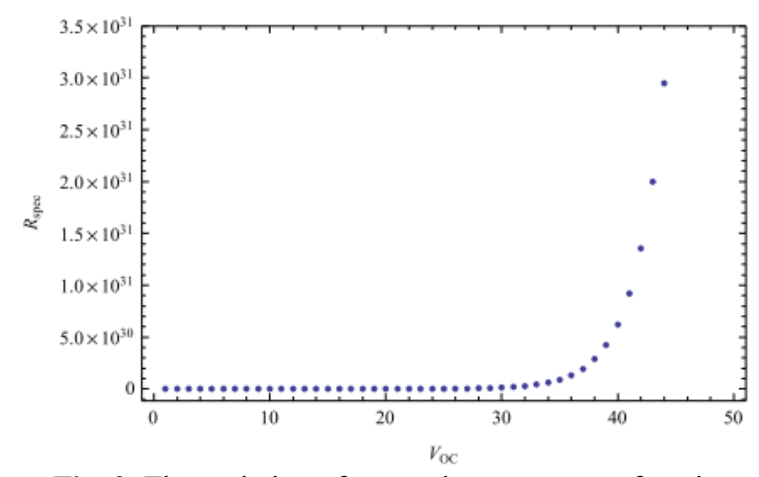

Fig. 2. The variation of spectral response as a function of open-circuit potential

The spectral response varies very slowly with the opencircuit potential up to a threshold value whereincreases very quickly, so that the spectral response is an exponential function of voc which in turn dependson the arithmetic mean of the valence band energy as well as the conduction band energy plus voc increases with increasing conduction band energy and decreasing valence band energy, which leads to an increase in the spectral response of the cell.

In reverse polarization, the current of the inversely polarized photovoltaic cell is:

$$
J_{\text {inv }}=J_{0}+J_{p h}(\lambda)
$$

This means

$$
J_{i n v}=q\left[\frac{n_{i}^{2} L_{h}}{N_{D} \tau_{h}}+Q_{E} \frac{\lambda}{h c} \Phi(\lambda)\right]
$$




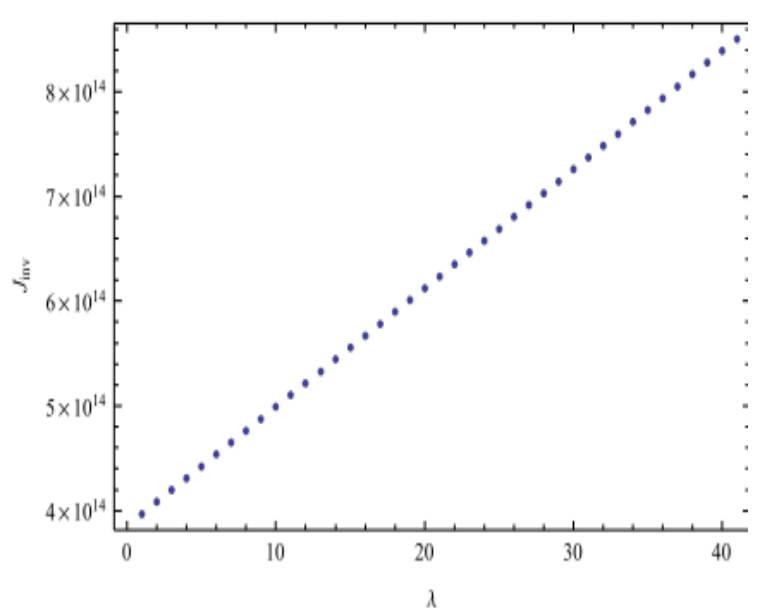

Fig. 3. The variation of the polarization inversion current density as a function of wavelength

This graph illustrates the variation of the polarization inversion current density with the wavelength of the incident light. It can be seen that this current density is an increasing function of the wavelength, that is to say that the polarization inversion current density is a function of the wavelength of the incident light, that is to say that the polarization inversion current density is an increasing function of the wavelength of the incident light. that is to say that the optimal

wavelengths for a current density of the photo induced charge carriers is in the visible range of the electromagnetic spectrum and more precisely the long wavelengths this could appear paradoxical but it is not the case of the fact that the polarization inversion current density depends more on the luminous flux than on the wavelength and this means that the current density depends on the photon numbers much more

than on the individual energy and this means that the photo current in turn then increases with the photon number with the luminous flux.

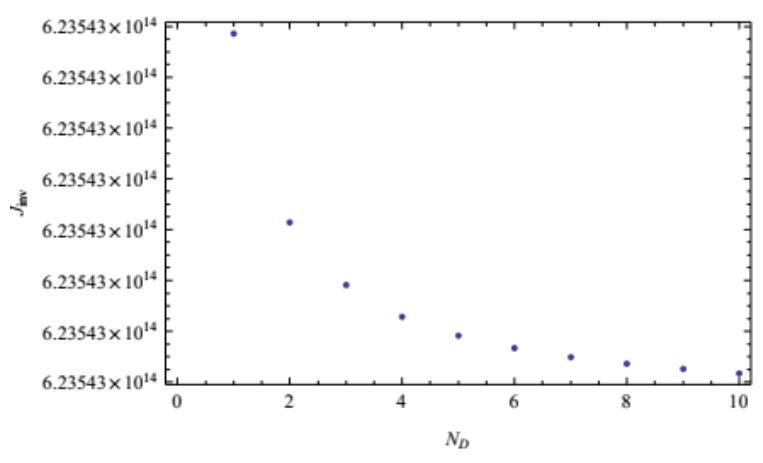

Fig. 1. Dependence of the polarization inversion current density and the density of donor impurities

According to the above graph we see that the density of the polarization inversion current decreases rapidly with the density of the donor impurity and this is due to the decrease of the saturation current density, which leads to the increase of the photo-current density with the density of the donor impurity. This can be explained by the fact that the width of the forbidden band decreases, thus the increase of the mobility of the photo induced charge carriers that constitute the photo-current, especially the part that is due to the transition of the electrons from the valence band to the conduction band.
If we account for the parallel variation of the polarization inversion current as a function of the density of the donor impurities and the luminous flux, we obtain the following 3D graph

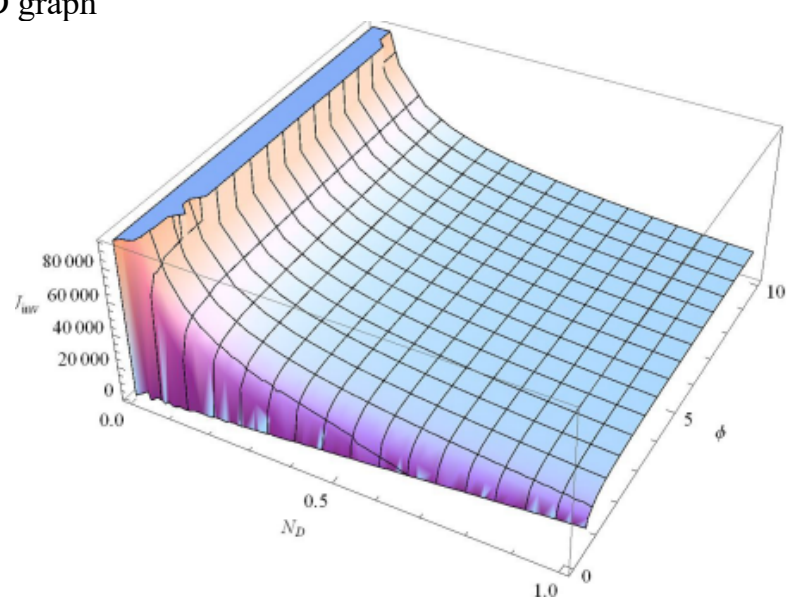

Fig. 2. the variation of $J_{\text {inv }}$ with the $N_{D}$ and the incident luminous flux

In this $3 \mathrm{D}$ graph it is constant that the polarization inversion current density decreases strongly with the density of the donor impurities which determines the saturation current density which becomes negligible in front of the photo current, that is to say that the polarization inversion current density depends in this case essentially on luminous flux and increases with its increase, and since $\mathrm{j}$ inv decreases with the donor doppage it leads to an increase of the photo current.

\subsection{Equivalent electrical circuit of the photovoltaic cell}

\subsubsection{Space charge modeling condenser:}

The capacity of SCZ is expressed by modeling it with a condenser as follows:

$$
C=\sqrt{\varepsilon S q N \frac{1}{[(\phi b i)-V]}}
$$

The thickness of the space charge zone depends strongly on the potential drop as well as the density of the donor and acceptor impurities this dependence is given by the equation

$$
\delta=\sqrt{2 \varepsilon \Delta \phi \frac{\left(\frac{1}{N A}-\frac{1}{N D}\right)}{q}}
$$




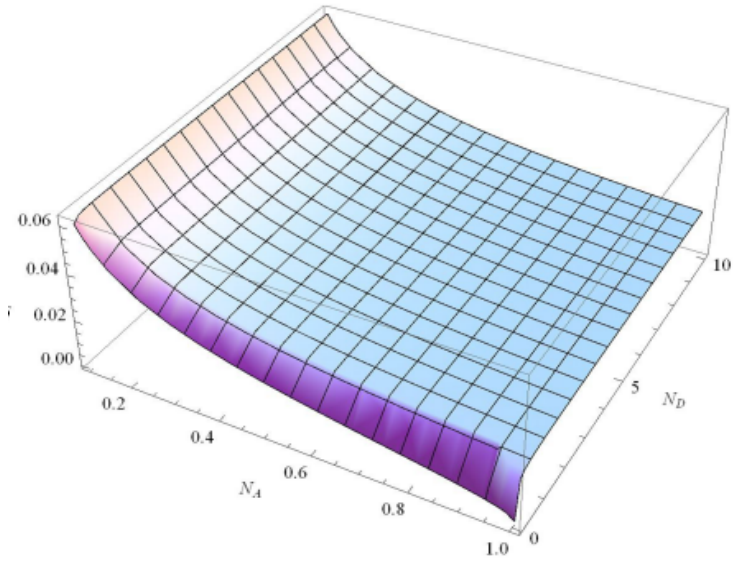

Fig. 3. The variation in the thickness of the space charge zone of the n-type and p-type doping

We can see from this $3 \mathrm{~d}$ graph that the thickness of the space charge zone decreases with type $\mathrm{P}$ doping and increases with type $\mathrm{n}$ dropping. This leads to an increase in the dissociation of the excitons by the increase of the electric field generated by the pn junction, which leads to an increase in the current generated by the space charge zone, which is due to the separation of the charges generated by the dissociation of the excitons.

\subsubsection{Solar cell modelling}

This cell can be assimilated to an R.L.C circuit where C represents the capacitance of the space charge zone the resistances $R=R_{s}+R_{L}+R_{s h}$ and the coil is assimilated to the polarization current this circuit can be described by the differential equation

$$
\mathbf{V}_{\mathbf{c}}+\mathbf{R C} \frac{\mathbf{d V}_{\mathbf{c}}}{\mathbf{d t}}+\mathbf{L C} \frac{\mathbf{d}^{2} \mathbf{V}_{\mathbf{C}}}{\mathbf{d t}^{2}}=\mathbf{E}
$$

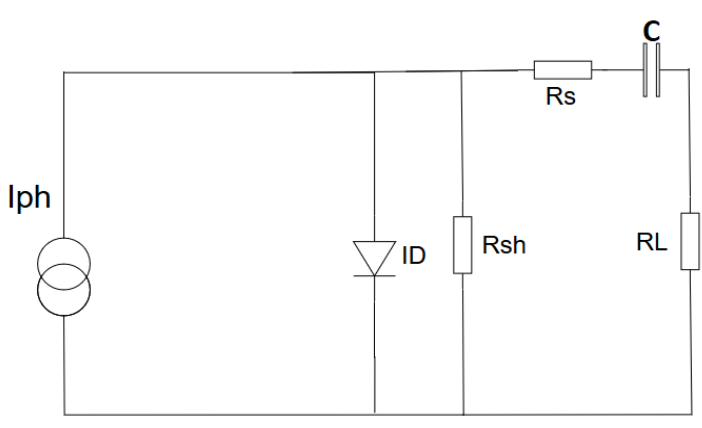

Fig. 4. Equivalent circuit of the solar cell studied

$$
Z=\sqrt{\left(\frac{1}{i C \omega}\right)^{2}+(i L \omega)^{2}+R^{2}}
$$

And the phase of the current has the form:

$$
\phi=\operatorname{Arctan}\left(\frac{\frac{1}{C \omega}-L \omega}{R}\right)
$$

For the recombination the time can be expressed as follows

$$
R_{R e c} C_{P E C(\operatorname{Rec})}=\tau_{R e c}
$$

For a bimolecular recombination we can write

$$
R_{\text {Rec }}=\frac{K_{A}}{C_{P E C(\operatorname{Rec})}}\left[\frac{q^{2} \Delta n\left[n_{e} n_{h}-n_{i}^{2}\right]\left(D_{e}+D_{h}\right)}{\varepsilon K T}\right]
$$

\section{Conclusion}

The modulation of the electronic properties of semiconductors, especially silicon, allows the optical and electrical characteristics of silicon-based photovoltaic cells to be optimized. This modulation is only possible by an optimal doping as well as by the control of the morphology and the thickness of the $p-n$ junction, which requires the implementation of laborious and expensive physico-chemical characterization techniques [15-16]. This is the reason why we have resorted to the electrical modeling of the photovoltaic cell by an RLC circuit as well as to the numerical simulation of some of its optical and structural characteristics (density of impurities) (input functions) in relation to the electrical characteristics of the cell (output function).

In future research, since we used numerical simulations to solve our problem, we can make use of the work of Daaif et al. [18] who programmed computer solutions to solve different manipulations of crystallography, thus by the development of virtual laboratories [19-20].

A multitude of mathematical and numerical techniques are possible, but we have opted for the finite difference method and the Runge-Kutta algorithm, which allows easy numerical resolution of drift diffusion and charge distribution equations that describe the essential transport properties of photo-induced charge carriers as well as the influence of space charge zone on the electrical properties of the cell, especially in relation to exciton dissociation. In this work, we have also drawn attention to the theoretical description of the physical phenomena characterizing the photovoltaic conversion, which allowed us to highlight the links between the electronic structure of silicon and the optical and electrical properties of the cell [17].

The electrical modeling allowed us to represent the cell by an equivalent electrical circuit modeling the different layers as well as the interfaces of the cell through three resistors, a capacitor as well as a diode and a coil; it also allowed us to model the physical processes on which the operation of the photovoltaic cell is based. By numerical simulation we tried to understand the relationship between the total current, the inversion current, and the optical parameters of the incident light such as luminous flux and wavelength, we also found a strong dependence of the polarization inversion current, and photocurrent as well as the thickness of the space charge zone and the density of impurities due to the donor doping which influences the current due to electrons and the acceptor doping which influences the current due to holes, the influence of the open circuit potential on the spectral response was also studied. 
To conclude with this work, which in no way claims to be exhaustive, we hope that it is a modest contribution to the theoretical study and modeling of silicon-based photovoltaic cells.

\section{Références}

1. Yang, M. M., Kim, D. J., \&Alexe, M. (2018). Flexo-photovoltaiceffect. Science, 360(6391), 904907.

2. S. ElMakhloufy, El .M. Majdi, A.Ouasri, S. Chtita, M. Saadi, L. Ammari, A. Cherqaoui, S. Belaaouad. Synthesis, crystal structure, IR, Ramanspectroscopy and DFT computation of monostrontium phosphate monohydrate, $\mathrm{Sr}(\mathrm{H} 2 \mathrm{PO} 4) 2 \cdot \mathrm{H} 2 \mathrm{O}$. Journal of Coordination Chemistry, 1-19, (2020).

3. Shabaev, A., Efros, A. L., \&Nozik, A. J. (2006). Multiexciton generation by a single photon in nanocrystals. Nano letters, 6(12), 2856-2863.

4. Ellingson, R. J., Beard, M. C., Johnson, J. C., Yu, P., Micic, O. I., Nozik, A. J., ... \&Efros, A. L. (2005). Highly efficient multiple exciton generation in colloidal $\mathrm{PbSe}$ and $\mathrm{PbS}$ quantum dots. Nano letters, 5(5), 865-871.

5. Li, G., Lu, Y., Xuan, Q., Akhlaghi, Y. G., Pei, G., Ji, J., \& Zhao, X. (2020). Small scale optimization in crystalline silicon solar cell on efficiency enhancement of low-concentrating photovoltaic cell. Solar Energy, 202, 316-325.

6. El Filali, B., Titov, O. Y., \&Gurevich, Y. G. (2018). Physics of charge transport in metalmonopolar (n-or p-type) semiconductor-metal structures. Journal of Physics and Chemistry of Solids, 118, 14-20..

7. S. Hamham, A. Cherqaoui, S. Belaaouad, et Y. Naimi, « Organic Semiconductivity and Photovoltaism: Concepts and applications », Mediterr.J.Chem., vol. 9, no 1, p. 65-77, août 2019, doi: 10.13171/mjc91190820600sh.

8. S. Hamham, S. Belaaouad, et Y. Naimi, « Physicochemical Characterization, Structuration and Morphology of Photo-active Heterojunction (P3htPcbm) Used In Organic Photovoltaic Cells »:, in Proceedings of the 1st International Conference of Computer Science and Renewable Energies, Ouarzazate, Morocco, 2018, p. 218-225, doi: $10.5220 / 0009776102180225$

9. YounessChaib, SoufianeZerraf, RafikBelghit, Mohammed Moutaabbid, Said Benmokhtar, Said Belaaouad. First-principles calculations on structural, electronic and magnetic properties of $\mathrm{V}$ doped GaAs semiconductor. https://doi.org/10.30534/ijeter/2020/100810202. ISSN 2347 - 398. Volume 8. No. 10, 7257-7261, October 2020

10. Benaichi, M., Chetouani, A., Karkri, A., Moussaid, D., \&Elqabbaj, S. E. (2019). ThreeDimensional Drift-Diffusion Model for Simulation and Investigation of Bordering Effects in Silicon
Solar Cells. Materials Today: Proceedings, 13, 630-636.

11. Chahid, E., Fedaoui, M., Nachaoui, M., Chowdhury, N. R., \&Malaoui, A. (2017).

Development of Numerical Method for Optimizing Silicon Solar Cell Efficiency.

12. Dadashbeik, M., Fathi, D., \&Eskandari, M. (2020). Design and simulation of perovskite solar cells based on graphene and $\mathrm{TiO}$ /graphene nanocomposite as electron transport layer. Solar Energy, 207, 917-924.

13. Zhao, J., Zhan, R., \& Xu, Y. (2020). Explicit exponential Runge-Kutta methods for semilinear parabolic delay differential equations. Mathematics and Computers in Simulation, 178, 366-381

14. Xia, J., Zou, J., Peng, X., Deng, W., \& Zhang, Y. (2020). Theoretical modeling and simulationbased assessment of graded-bandgap $\mathrm{AlGaAs} / \mathrm{GaAs}$ electron-injection cathode. Ultramicroscopy, 113121.

15. Mambrini, T. (2014). Caractérisation de panneaux solaires photovoltaïques en conditions réelles d'implantation et en fonction des différentes technologies (Doctoral dissertation, Paris 11).

16. M. TRIDANE, A. KHEIREDDINE, I. FAHIM, H. MOUTAABBID, M. MOUTAABBID, EL. M. TACE, A. CHARAF, M. RADID, F. SALHAMEN, S. BENMOKHTAR AND S.BELAAOUAD.Chemical preparation, crystallographic characterization and vibrational study of two new condensed phosphates. Verres, Céramiques \& Composites, Vol. 4, n 1, 20-27 (2015).

17. El Mahdi MAJDI, Soufiane ZERRAF, Hamza MAROUANI, Smail EL MAKHLOUFI, Mustapha BELHABRA, Ali OUASRI, Youssef MAIMI and Said BELAAOUAD. « Structural and Vibrational Study of Titanium Monophosphates $\mathrm{Na} 0.5 \mathrm{M} 0.25 \mathrm{Ti} 2$ (PO4)3 (M= Mn, Ni) »Mediterranean Journal of Chemistry (MJC). Volume-9 Issue-5, November 2019, pp (355-362). DOI : $10.12171 / \mathrm{mjc} 01911281083 \mathrm{emm}$

18. Daaif J., Zerraf S., Tridane M., Benmokhtar S., Belaaouad S. (2019) Technological Innovation in Teaching and Research in Chemical Science: Development of a Computer Application for the Simulation of the Practical Works of Crystallography. International Journal of Recent Technology and Engineering. Volume-8 Issue-3,; 2399-2405. DOI: 10.35940 / ijrte.C4665.098319.

19. Daaif J., Zain S., Zerraf S., Tridane M., Benmokhtar S., Belaaouad S. (2019). Progress of Digital Learning Resources: Development and Pedagogical Integration of a Virtual Environment Laboratory for the Practical Experiments in Chemistry. International Journal of Innovative Technology and Exploring Engineering. Volume-8 Issue-11; 4239- 4245. DOI: 10.35940 / ijitee.K2403.0981119. 
20. Daaif J., Zerraf S., Tridane M., Benmokhtar S., Belaaouad S. (2019). Pedagogical engineering to the teaching of the practical experiments of chemistry: Development of an application of three dimensional digital modelling of crystalline structures. Cogent

Education, 2019, 6(1), 1708651. DOI :

https://doi.org/10.1080/2331186X.2019.1708651 